\title{
3-D recursive extrapolation operators: an overview
}

\author{
J.W. Thorbecke and A.J. Berkhout \\ Centre for Technical Geoscience, \\ Laboratory of Seismics and Acoustics,

\section{Summary} \\ Extrapolation through 3-Dimensional inhomogeneous media is a computer \\ intensive operation. Recently various authors have published articles which \\ pay attention to an optimized calculation and efficient implementation of \\ 3-Dimensional extrapolation operators. This paper gives an overview of the \\ existing methods and introduces an efficient optimization method that has \\ not yet been discussed.
}

\section{Introduction}

In laterally homogeneous media the recursive one-way extrapolation operator in the $k_{x}, k_{y}-\omega$ (wavenumber-frequency) domain can be well approximated by the simple phase shift operator (Gazdag [3]):

$$
\tilde{F}\left(k_{x}, k_{y}, \omega, \Delta z\right)=\exp \left(j \sqrt{\frac{\omega^{2}}{c^{2}}-\left(k_{x}^{2}+k_{y}^{2}\right)} \Delta z\right)
$$

with $\Delta z$ being a small extrapolation step. The advantage of computation in the $k_{x}, k_{y}-\omega$ domain is that the desired result is obtained by multiplication of the data with the operator. However, to allow laterally varying medium functions a space variant convolution operator in the $x, y$ - $\omega$ (space-frequency) domain should be applied. When the spatial extrapolation operator is used in an explicit recursive depth migration algorithm it must be calculated in such a way that it gives reliable and stable results within a reasonable computation time.

A first subdivision between different optimization methods can be made with respect to the type of expansion of the analytical phase shift operator (equation (1)) in the wavenumber domain. This expansion can be a Taylor series with respect to $k_{z}=\sqrt{k^{2}-\left(k_{x}^{2}+k_{y}^{2}\right)}$ (this paper), a modified Taylor series with specialized basis functions (Hale [5]) or an expansion with respect to $k_{x}^{2}+k_{y}^{2}$ (Berkhout [1], Soubaras [9] and this paper). The phase shift operator can also be approximated by a Chebyshev polynomial (Soubaras 

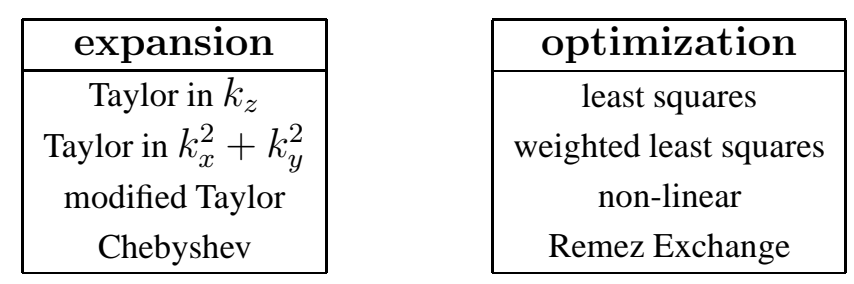

\begin{tabular}{|c|}
\hline implementation \\
\hline 3-D convolution \\
2-D convolution \\
McClellan \\
1-D convolution \\
\hline
\end{tabular}

Table 1: Three criteria which can be used to discriminate between different 3 -D recursive extrapolation operators.

[9]). A second subdivision can be made with respect to the kind of optimization method used to obtain the spatial convolution operator. The type of implementation of the 2-Dimensional spatial convolution is a third criterion to discriminate between different methods. Table 1 gives an overview of the different techniques which can be used in the expansion, optimization or implementation method.

For example Holberg [6] and Blacquière [2] use a non-linear optimization technique for the operator optimization and have implemented this operator as a full 2-D convolution. Hale [4] makes use of the McClellan transformation and can use rotated 1-D convolution operators. Soubaras [9] uses the Remez exchange algorithm in the optimization of the 1-D convolution operators and in the optimization of the expansion factors (with respect to powers of $k_{x}^{2}+k_{y}^{2}$ ) of the phase shift operator. In this paper two different expansions of the analytical extrapolation operator are discussed and the weighted least squares optimization method is introduced as a fast alternative optimization. The weighted least squares technique can be used in the optimization of the convolution operators and in the optimization of the expansion factors.

\section{Theory of weighted least-squares}

The most simple way to obtain space-frequency operators is to transform the exact operators from the wavenumber-frequency domain back to the spacefrequency domain with the aid of a Fourier transform. Despite the simple form of the solution it is not very efficient because the spatial convolution operator obtained in this way must be very lengthy to give stable and accurate results. Tapering the spatial operator gives some improvements (Nautiyal [7]) but for accurate extrapolation results tapering cannot be used [10]. The aim in the design of the operator is a short convolution operator with a wavenumber-frequency spectrum which is, over a desired wavenumber band, equal or close to the phase shift operator in the $k_{x}, k_{y}-\omega$ domain. For 1Dimensional operators (in 2-Dimensional media) this problem is simple and can be written in the following matrix notation

$$
\overrightarrow{\vec{F}^{\prime}}=\Gamma \vec{F} \text { or, }
$$




$$
\tilde{F}^{\prime}\left(n \Delta k_{x}\right)=\Delta x \sum_{m=-M}^{M} F(m \Delta x) \exp \left(j m \Delta x n \Delta k_{x}\right)
$$

with $2 M+1$ being the user specified length of the desired short operator and $\tilde{F}^{\prime}$ being its spatial Fourier transform, yielding an approximation of the exact phase shift operator. Equation (2) has to be solved for the unknown operator coefficients $\left.F_{m}=F(m \Delta x)\right)$ for all wavenumbers of interest. A weighted error function $\tilde{\varepsilon}$ is defined as

$$
\begin{aligned}
\tilde{\varepsilon} & =\overrightarrow{\tilde{E}^{h}} \tilde{\Lambda} \overrightarrow{\tilde{E}} \text { with } \\
\overrightarrow{\tilde{E}} & =\Gamma \vec{F}-\overrightarrow{\tilde{F}}
\end{aligned}
$$

and $\tilde{\Lambda}$ a diagonal matrix containing the weighting function on its diagonal. By introducing this weighting function we have a good control over the desired functionality of the space-frequency operators. The least-squares solution of equation (4) is given by

$$
\vec{F}=\left[\Gamma^{h} \tilde{\Lambda} \Gamma\right]^{-1} \Gamma^{h} \tilde{\Lambda} \overrightarrow{\tilde{F}}
$$

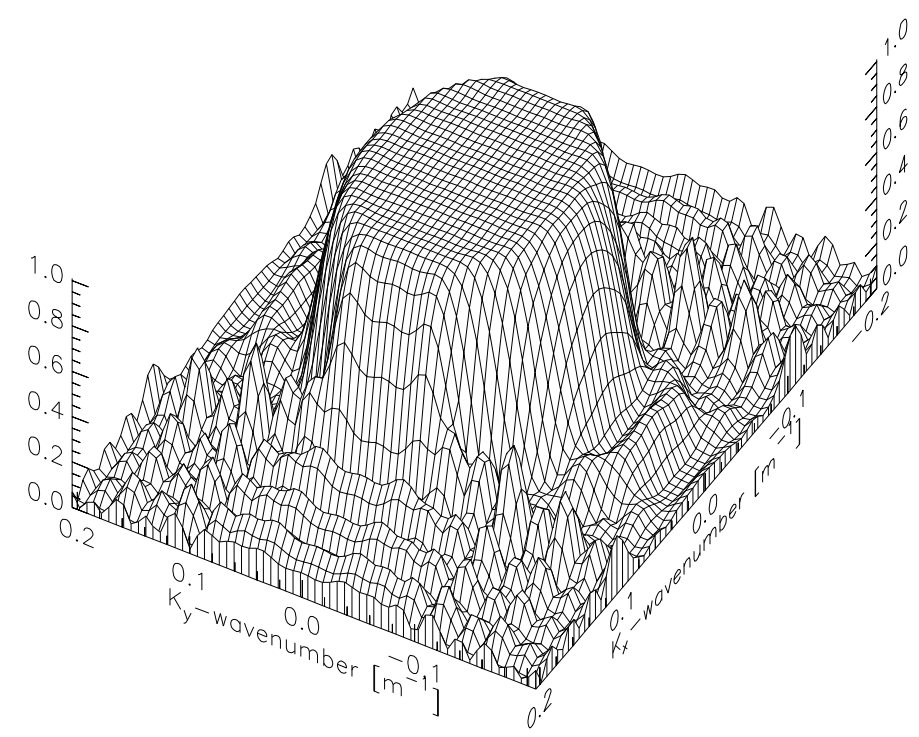

Figure 1: Wavenumber spectrum obtained with direct optimization using the weighted least-squares method. Note that the spectrum is close to the spectrum of the exact phase shift operator for the chosen band of interest $\left(k_{x}^{2}+k_{y}^{2}<k^{2} \sin ^{2}\left(\alpha_{\max }\right)\right.$ with $\left.\alpha_{\max }=65^{\circ}\right)$.

Note that the matrix $\Gamma^{h} \tilde{\Lambda} \Gamma$ has a Toeplitz structure and can be inverted fast by using a Levinson scheme. 
In the following sections three different methods; direct, expansion in $k_{z}$ and expansion in $k_{x}^{2}+k_{y}^{2}$ are discussed. In each of these methods the weighted least-squares (WLSQ) method can be used. In a direct optimization for the 2-D operators the fast Toeplitz inversion can be used by optimizing $k_{x}$ to $x$ and successively $k_{y}$ to $y$.

\section{Direct method}

The direct method does not make use of a series expansion. The optimization for the convolution operator is directly done in the transformation from wavenumber to spatial domain by means of an optimization scheme (see Holberg [6] or Blacquière [2] for the results using a non-linear optimization scheme). A disadvantage of this direct method is that in the space-frequency domain the full 2-D convolution has to be carried out for every spatial position. For a typical operator with $c=2000 \mathrm{~ms}^{-1}, f=30 \mathrm{~Hz}, \Delta x=\Delta y=$ $\Delta z=15 \mathrm{~m}$ and a maximum angle of interest $\left(\alpha_{\max }\right)$ of $65^{\circ}$ the operator length is typically $19 \times 19$ points which means 361 multiplications and summations for every grid point! In Figure 1 the wavenumber spectrum of our linear WLSQ optimized, $19 \times 19$ points, $65^{\circ}$ spatial convolution operator is given for $256 \times 256 k_{x}, k_{y}$ points (in the remainder of this paper these parameters will be used in all examples). From Figure 1 we see that the WLSQ method gives a stable and accurate operator which has a wavenumber spectrum close to the exact phase shift operator.

\section{Single series expansion}

The phase-shift operator can be written in a Taylor expansion with respect to $k_{z}$. By truncating this series expansion the operator is approximated by a polynomial in $k_{z}$, according to

$$
\exp \left(j k_{z} \Delta z\right) \approx 1+j \Delta z k_{z}+\frac{(j \Delta z)^{2}}{2 !}\left(k_{z}\right)^{2}+\mathrm{O}\left(k_{z}\right)^{3}
$$

The number of terms in the expansion depends on the accuracy required and the maximum angle of interest. The coefficients in the series expansion can be obtained by using the constants in the Taylor series as given in equation (7) or by using an optimization technique. In Figure 2 the wavenumber spectrum of the approximated phase shift operator is shown for a sixth order series expansion with optimized coefficients for the exact $k_{z}$ operators (the same parameters are used as in the example for the direct method). Outside the band of interest $\left(\alpha_{\max }=65^{\circ}\right)$ the exact $k_{z}$ operator is set to zero. Note that with six terms there is already a good match with the analytical spectrum. 


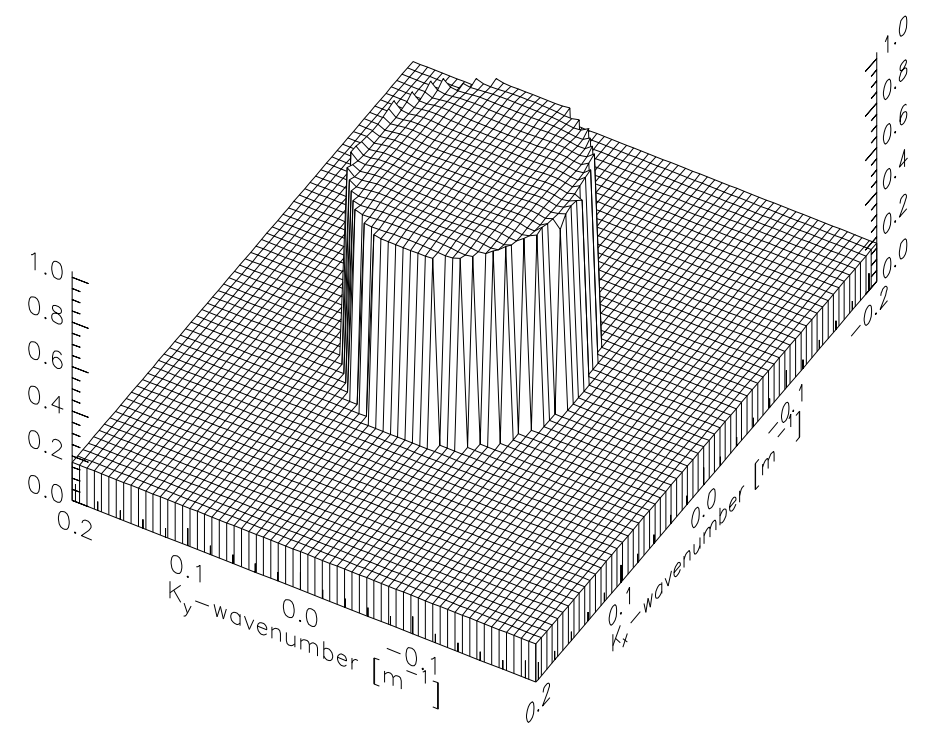

Figure 2: Wavenumber spectrum of the approximated phase shift operator for a sixth order series expansion with WLSQ optimized $a_{n}$ coefficients for the exact $k_{z}$ operators, taking $k_{z}=0$ for $\alpha>65^{\circ}$.

To arrive at the spatial convolution scheme, which is given in Figure 3, the $k_{z}$ operator is transformed in some optimum way to the space domain and applied several times to the data. Every time a 2-D convolution (indicated by the box $o p(x, y)$ ) with the spatial $k_{z}$ operator is carried out on the data, a new term is added to the series expansion. It is interesting to note that an exact analytical expression for $o p(x, y)$ can be derived (see Berkhout [1], appendix E). However, a weighted least-squares version yields a shorter operator. Note that the factors $a_{n}$ in Figure 3 can also be obtained by a least-squares optimization with respect to the wavenumber spectrum of the optimized $k_{z}$ operator. The WLSQ optimization of the spatial $k_{z}$ convolution operator itself results in a short operator which is shown in Figure 4 for a

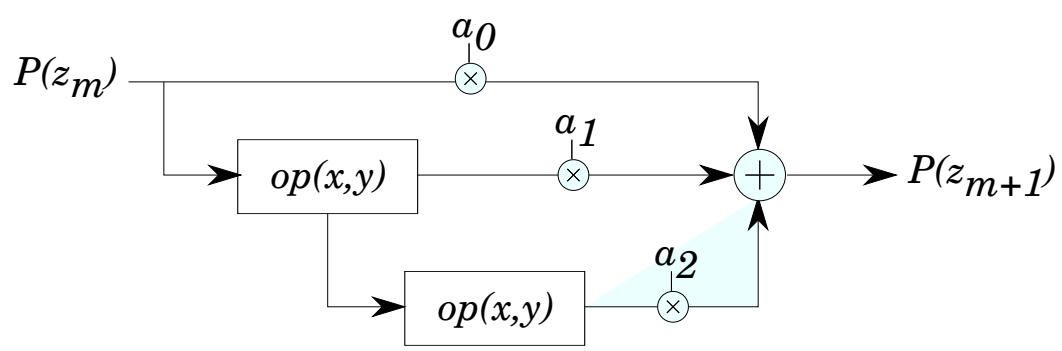

Figure 3: Spatial convolution scheme for a series expansion in $k_{z}$. Here a second-order scheme is shown. 


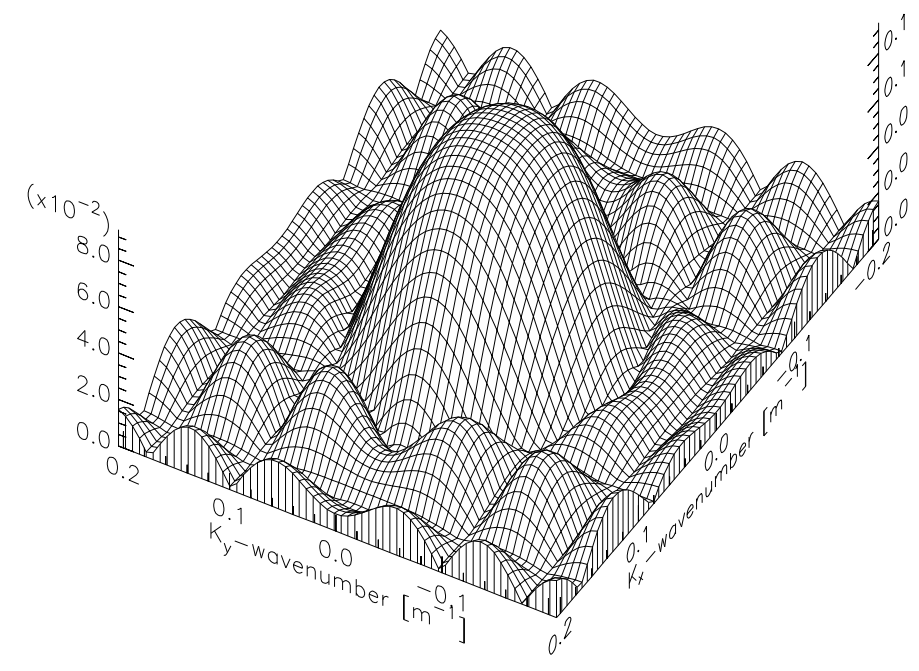

Figure 4: Spectrum of a $7 \times 7$ WLSQ optimized $k_{z}$ convolution operator $\left(\alpha_{\max }=65^{\circ}\right)$.

$7 \times 7$ operator. Using this 2 -D convolution operator the computational effort can be reduced in comparison with the direct method. For example if we take six terms in the expansion there are $5 \times(7 \times 7)+6=251$ multiplications and additions needed for every grid point. During the presentation of this paper we will demonstrate how many terms are needed for a stable and accurate operator. In Figure 5 the wavenumber spectrum of the approximated operator is shown for a series expansion up to the 6'th order and with a $7 \times 7$ basic convolution operator. This spectrum is not the best possible solution, but it shows the basic principle of the method. The wavenumber spectrum for $k_{y}=0$ and the amplitude error are shown in Figure 6 for an operator with 3 (dotted line),6 (dashed line) and 9 (solid line) terms in the series expansion with WLSQ optimized coefficients for the WLSQ optimized $k_{z}$ operator.

\section{Double series expansion}

By using an additional series expansion, i.e. $k_{z}$ is expanded in terms of $k_{x}^{2}+k_{y}^{2}$, there is an extra advantage (Berkhout [1], chapter 10). The basic spatial convolution operators are reduced to simple 1-D convolution operators: $d_{2}(x)$ and $d_{2}(y)$. The series expansion in $k_{x}^{2}+k_{y}^{2}$ is given in equation (8):

$$
\begin{gathered}
\exp \left(j k_{z} \Delta z\right) \approx 1- \\
\frac{j \Delta z}{2 k}\left(k_{x}^{2}+k_{y}^{2}\right)- \\
\frac{j \Delta z}{8 k^{3}}(1-j k \Delta z)\left(k_{x}^{2}+k_{y}^{2}\right)^{2}-
\end{gathered}
$$




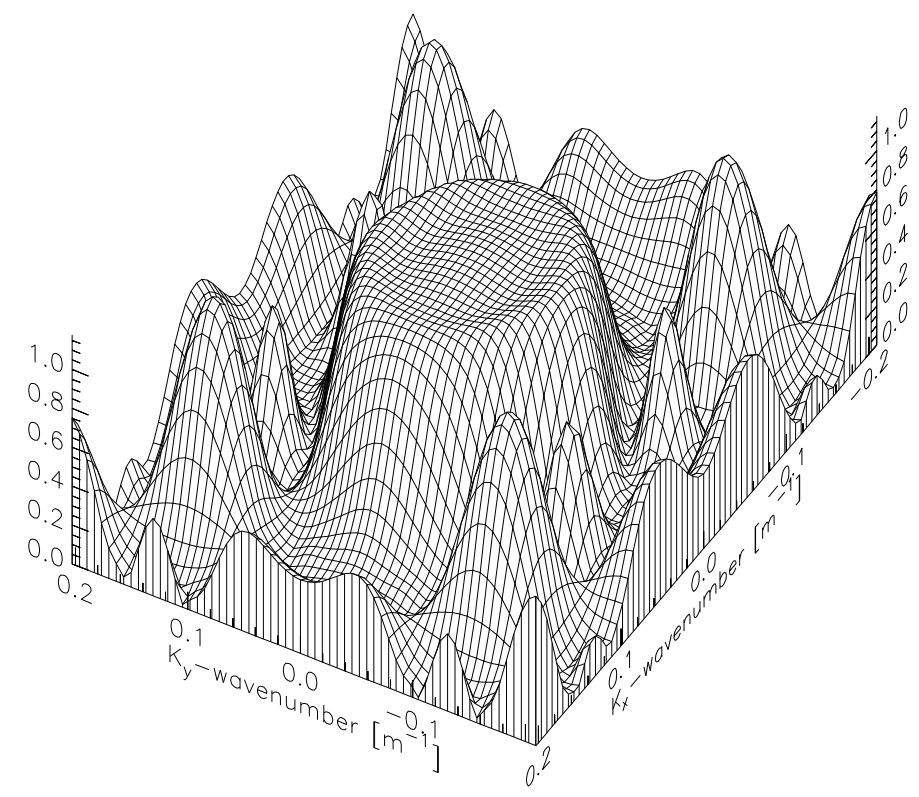

Figure 5: Wavenumber spectrum of the approximated phase shift operator for a sixth order series expansion with optimized coefficients for the WLSQ optimized $k_{z}$ operators $\left(\alpha_{\max }=65^{\circ}\right)$.

$$
\begin{gathered}
\frac{j \Delta z}{16 k^{5}}\left(1-j k \Delta z+\frac{(j k \Delta z)^{2}}{3}\right)\left(k_{x}^{2}+k_{y}^{2}\right)^{3}+ \\
\mathrm{O}\left(\left(k_{x}^{2}+k_{y}^{2}\right)^{4}\right)
\end{gathered}
$$

The same techniques as discussed in the series expansion in $k_{z}$ can be used again: the spatial versions of $k_{x}^{2}$ and $k_{y}^{2}$, i.e. $d_{2}(x)$ and $d_{2}(y)$, are determined by a weighted least-squares process (see Figure 7 ). Now the computational effort is reduced even more (Figure 8). For example using six terms in the expansion and a 7 point convolution operator leads to $5 \times(7+7)+6=76$ multiplications and additions for one grid point. Soubaras [9] used the same type of expansion in $k_{x}^{2}+k_{y}^{2}$, but the optimization technique for both the terms in the series expansion and the convolution operators is the Remez exchange algorithm. Sollid [8] uses the same expansion with least squares optimization and a McClellan implementation for the convolution.

\section{Discussion and Conclusions}

The weighted least-squares optimization method is an efficient procedure which gives stable and accurate operators [10]. By using a series expansion of the phase shift operator it is possible to reduce the computation time in comparison with a direct 2-Dimensional convolution. Two expansions were discussed in this paper: one in $k_{z}$ and one in $k_{x}^{2}+k_{y}^{2}$. From an efficiency point of view the expansion in $k_{x}^{2}+k_{y}^{2}$ appears the most interesting one. 

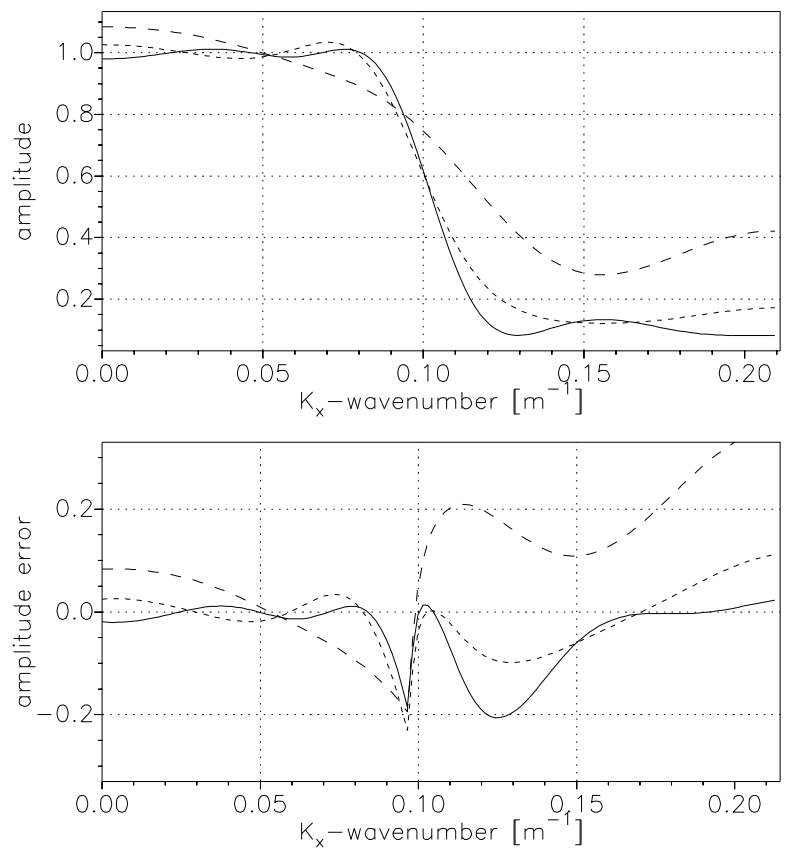

Figure 6: Amplitude and amplitude error for the slice $k_{y}=0$ of the approximated phase shift operator for a 3th, 6th and 9th order series expansion with WLSQ optimized coefficients for the WLSQ optimized $k_{z}$ operators.

Two levels of optimization are carried out: one with respect to the operators $\left(k_{z}^{2}\right.$ or $k_{x}^{2}$ and $\left.k_{y}^{2}\right)$ and one with respect to the expansion factors $\left(a_{n}\right)$.During the presentation we will compare the impulse responses and the computational effort of the weighted least squares optimization with the Remez exchange algorithm.

\section{Acknowledgement}

This research has been carried out at the Laboratory of Seismics and Acoustics of the Delft University of Technology as a part of the Delphi-consortium. We wish to thank the sponsors of the consortium for their financial support and their continuing interest in this research project.

\section{References}

[1] A. J. Berkhout. Imaging of acoustic energy by wave field extrapolation (2nd edition); chapter 10, appendix E. Elsevier Amsterdam, 1982.

[2] G. Blacquière, H. W. J. Debeye, C. P. A. Wapenaar, and A. J. Berkhout. 3D table-driven migration. Geophysical Prospecting, 37:925958, November 1989. 

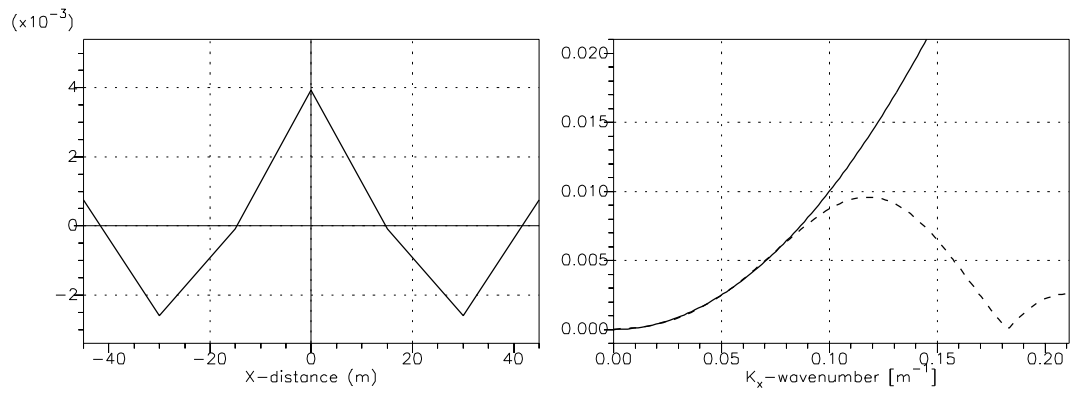

Figure 7: Optimized 7 point $d_{2}(x)$ operator (left) and its wavenumber spectrum (right), chosing $\alpha_{\max }=65^{\circ}$. The dotted line represents the spectrum of the WLSQ optimized operator and the solid line indicates the exact $k_{x}^{2}$ function.

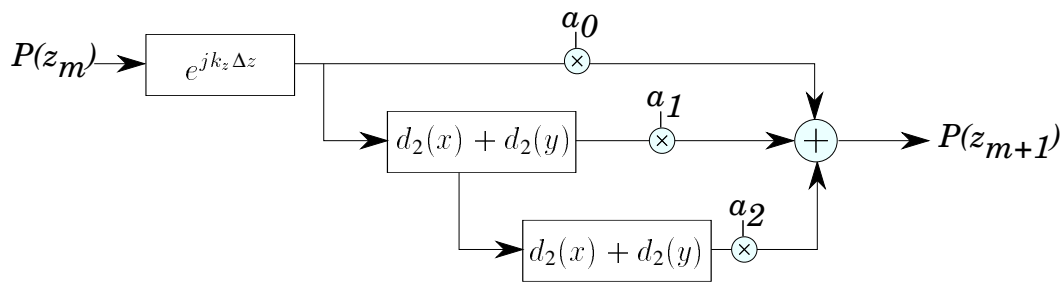

Figure 8: Spatial convolution scheme for a double series expansion in $k_{x}^{2}+k_{y}^{2}$. Here a second-order scheme is shown.

[3] J. Gazdag. Wave equation migration with the phase-shift method. Geophysics, 43:1342-1351, 1978.

[4] D. Hale. 3-D depth migration via McClellan transformations. Geophysics, 56:1778-1785, November 1991.

[5] D. Hale. Stable explicit extrapolation of seismic wavefields. Geophysics, 56:1770-1777, November 1991.

[6] O. Holberg. Towards optimum one-way wave propagation. Geophysical Prospecting, 36:99-114, February 1988.

[7] A. Nautiyal, S. H. Gray, N. D. Whitmore, and J. D. Garing. Stability versus accuracy for an explicit wavefield extrapolation operator. Geophysics, 58:277-283, February 1993.

[8] A. Sollid and B. Arntsen. Wavefield extrapolators for cost-effective 3D depth migration. In Expanded Abstracts, page C046. EAEG, 1993. 
[9] R. Soubaras. Explicit 3-D migration using equiripple polynomial expansion and laplacian synthesis. In Expanded Abstracts, pages 905-908. SEG, 1992.

[10] J. W. Thorbecke and W. Rietveld. Optimum extrapolation operators: a comparison. In Expanded Abstracts, 2pp, EAEG, 1994. 